\title{
REFERENCES
}

1. J. L. Doob, Stochastic processes, New York, Wiley, 1953.

2. Nelson Dunford and J. T. Schwartz, Convergence almost everywhere of operator averages, J. Rational Mech. Anal. vol. 5 (1956) pp. 129-178.

3. - Linear operators, part 1, New York, Interscience, 1958.

4. L. Fejér, La convergence sur son cercle de convergence d'une série de puissance effectuant une représentation conforme du cercle sur le plan simple, C. R. Acad. Sci. Paris vol. 156 (1913) pp. 46-49. 1950.

5. J. von Neumann, Functional operators, vol. 2, Princeton University Press,

UNIVERSITY OF ILLINOIS AND

IBM Research Center, Yorktown Heights, New York

\section{A THEOREM ON OVERCONVERGENCE}

\section{F. SUNYER I BALAGUER}

The conjecture announced by A. J. Macintyre $[2 ; 3]$ is equivalent to the theorem stated and proved below.

TheOREM. Let $D$ be an open domain containing the origin and let $f(z)$ be a function regular in $D$ with the expansion $f(z)=\sum_{0}^{\infty} c_{n} z^{n}$. Let $D_{1}$ be a bounded closed domain contained in $D$. Then there exists a positive number $\lambda_{0}=\lambda_{0}\left(D, D_{1}\right)$ such that if $c_{n}=0$ for a sequence of intervals $n_{k} \leqq n \leqq \lambda n_{k}$ with $\lambda>\lambda_{0}$, then the subsequence of partial sums $s_{n_{k}}$ $=\sum_{0}^{n_{k}} c_{n} z^{n}$ converges uniformly to $f(z)$ in $D_{1}$.

Proof. Let $C D$ and $C D_{1}$ denote the complements of $D$ and $D_{1}$ respectively and let $h_{i}, i=1,2, \cdots$, be the components of $C D_{1}$. The components can be considered as disjoint and there exists only one unbounded component. The one unbounded component will be denoted as $h_{1}$.

One can assert that there exists only a finite number of components $h_{i}$ such that

$$
h_{i} \cap C D \neq \varnothing,
$$

where $\varnothing$ is the empty set. This assertion is proved as follows. Assume that there exists an infinite number of components $h_{i}, i \geqq 2$, such that (1) is valid. A bounded sequence of points $a_{i}$ can be formed where $a_{i} \in h_{i} \cap C D, i \geqq 2$. Every $a_{i}$ is an element of $C D$ and hence the dis-

Received by the editors June 27, 1960. 
tance $d$ from $D_{1}$ is at least $\delta>0$. The limit point $a$ of the sequence then must be such that $d\left(a, D_{1}\right) \geqq \delta>0$. Thus $a$ is an element of $C D_{1}$ and all points $z$ in $|z-a|<\delta$ must be in the same component.

Let the finite number of components be enumerated as $h_{i}, i=1,2$, $\cdots, N$. Considering now

$$
D_{2}=D_{1}+\bigcup_{i=N+1}^{\infty} h_{i}
$$

then $D_{2}$ is a bounded closed domain and $D_{2} \subset D_{1}$. Since $\sum_{i=N+1}^{\infty} h_{i}$ is bounded and $D_{1}$ is bounded by hypothesis, $D_{2}$ is bounded. Also, since $h_{i} \cap C D=\varnothing, i \geqq N+1$, then $h_{i} \subset D$ and $D_{2}$ is contained in $D$. To prove that $D_{2}$ is closed note that its complement is $\sum_{i=1}^{N} h_{i}$ and is open.

Now $N-1$ polygonal $\operatorname{arcs} L_{1}, L_{2}, \cdots, L_{N-1}$ can be chosen such that $D_{2}-\sum_{1}^{N-1} L_{k}$ is simply connected. Also $N-1$ other polygon $\operatorname{arcs} L_{1}^{\prime}, L_{2}^{\prime}, \cdots, L_{N-1}^{\prime}$ can be so chosen that $L_{k} \cap L_{j}^{\prime}=\varnothing$ and $D_{2}-\sum_{1}^{N-1} L_{k}^{\prime}$ is simply connected. Consider now the open circle $C(s, R)$ or $|z-s|<R$ and let $S(L, R)=U_{s \in L} C(s, R)$. Thus $S(L, R)$ is a strip enclosing the polygonal arc $L$. For $R$ sufficiently small,

$$
S\left(L_{k}, R\right) \cap S\left(L_{j}^{\prime}, R\right)=\varnothing .
$$

Hence for $R$ sufficiently small two closed simply connected domains can be defined, $D_{3}=D_{2}-\cup_{1}^{N-1} S\left(L_{k}, R\right)$ and $D_{3}^{\prime}=D_{2}-U_{1}^{N-1} S\left(L_{j}^{\prime}, R\right)$ such that $D_{3}+D_{3}^{\prime}=D_{2}$. This follows from

$$
\begin{aligned}
D_{3}+D_{z}^{\prime} & =D_{2}-\left\{\bigcup_{k=1}^{N-1} S\left(L_{k}, R\right)\right\} \cap\left\{\bigcup_{j=1}^{N-1} S\left(L_{j}^{\prime}, R\right)\right\} \\
& =D_{2}
\end{aligned}
$$

by (3).

The proof of the theorem follows. An open bounded simply connected domain $\Delta=\Delta\left(D, D_{3}\right)$ can now be defined such that $D_{3} \subset \Delta$, $\{|z|<r\} \subset \Delta, \bar{\Delta} \subset D$ where $r$ is the radius of convergence of $f(z)$ $=\sum_{0}^{\infty} c_{n} z^{n}$ and $\bar{\Delta}$ is the closure of $\Delta$. From the Nevanlinna two-constant theorem, if $F(z)$ is regular in $\Delta$

$$
M(\Delta)=\underset{z \in \Delta}{\operatorname{l.u.b}}|F(z)|, \quad M(d)=\underset{|z|<r / 2}{\text { l.u.b. }}|F(z)|,
$$

then [1]

$$
M\left(D_{z}\right)=\underset{z \in D_{z}}{\operatorname{l.u.b.}}|F(z)| \leqq\{M(\Delta)\} \cdot\{M(d)\}^{1-\theta}
$$

where $\theta>0$ depends on $D_{3}$ and $\Delta$. Using the majorization of $r_{n_{k}}$, where $r_{n_{k}}=f(z)-s_{n_{k}}, r_{n_{k}}=\sum_{n k}^{\infty} c_{n} z^{n}$. if $n_{k}$ is large we get l.u.b.|z|<r/2 $\left|r_{n_{k}}\right|<$ 


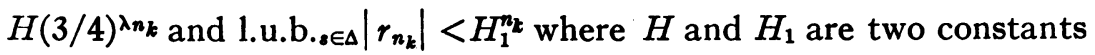
depending only on $\Delta$. Thus by (4),

$$
\text { 1.u.b. }\left|r_{n_{k}}\right| \leqq H^{1-\theta}\left\{B_{1}^{\theta}(3 / 4)^{\lambda(1-\theta)}\right\}^{n_{k}} \text {. }
$$

Thus if $\lambda>\lambda_{0}\left(\Delta, D_{3}\right)$ there is overconvergence in $D_{3}$. Similarly there is overconvergence in $D_{3}^{\prime}$ if $\lambda>\lambda_{0}\left(\Delta, D_{3}^{\prime}\right)$. Now since $D_{3}+D_{3}^{\prime}=D_{2}$ $\supset D_{1}$ the theorem is proved.

REMARK. By the same method similar results are proved for the series of Dirichlet and for the integral of Laplace.

\section{REFERENCES}

1. G. Bourion, L'ultraconvergence dans les séries de Taylor, Paris, Hermann, 1937.

2. A. J. Macintyre, Length of gaps and size of region of overconvergence. Preliminary report, Abstract 557-27, Notices Amer. Math. Soc. vol. 6 (1959) p. 186.

3. - Size of gaps and region of overconvergence, Collect. Math. vol. 11 (1959) pp. 165-174.

University of Barcelona, Spain 